\section{Kantonsarzt Hans Binz}

Die Revision des eidgenössischen Epidemiegesetzes 1970 verpflichtete die Kantone, einen Kantonsarzt einzusetzen. Diese sind sozusagen das Produkt einer Seuchenverordnung, wobei sich ihre Aufgaben und Kompetenzen in unserem föderalen System stark unterscheiden. In 17 Kantonen wird diese Funktion inzwischen vollamtlich wahrgenommen, in 9 Kantonen teilzeitlich, überwiegend neben einer Praxistätigkeit. Als Amtsärzte besprechen sie ihre gemeinsamen Anliegen regelmässig in der Vereinigung der Schweizerischen Kantonsärzte/-ärztinnen, zurzeit unter dem Präsidium von Hans Binz, Kantonsarzt von Solothurn.

Herr Binz, würden Sie uns bitte etwas über Ihren persönlichen und beruflichen Werdegang erzählen?

Ich bin am 5. September 1944 in Riedholz, Solothurn, geboren. Vater von 5 Söhnen. Meine Hobbies sind Klavierspielen, am liebsten Mozart und Beethoven, und die Beschäftigung mit Mineralien und Versteinerungen aus dem Jura. Ich bewege mich beim Radfahren, Klettern und auf Skitouren und lese zurzeit jeden Abend im «Zauberberg» von Thomas Mann.

Das Medizinstudium in Bern und Zürich habe ich 1971 an der Medizinischen Fakultät in Zürich abgeschlossen. 1971-1976 war ich Assistent am Institut für Medizinische Mikrobiologie an der Universität Zürich und habe am National Institute für Medical Research in Mill Hill, London, am Wallenberglaboratorium in Uppsala, Schweden, und am Institut für Immunologie an der Universität Uppsala gearbeitet. 1975 folgte die Ernennung zum Dozenten für Immunologie an der Universität Uppsala. 1976 war ich Oberassistent am Institut für Virologie und Immunologie der Universität Zürich und wurde 1979 Privatdozent für Immunologie. 1981 folgte die Assistenzprofessur für Immunologie und 1987 die Titularprofessur an der Universität Zürich. 1988 wurde ich als Forschungsdirektor an das Centre d'Immunologie Pierre-Fabre in Castres, Frankreich, gewählt. 1990-1997 war ich Direktor des Centre d'Immunologie Pierre-Fabre in Saint-Julien-en-Genevois, Frankreich. Seit Januar 1997 bin ich Kantonsarzt des Kantons Solothurn.

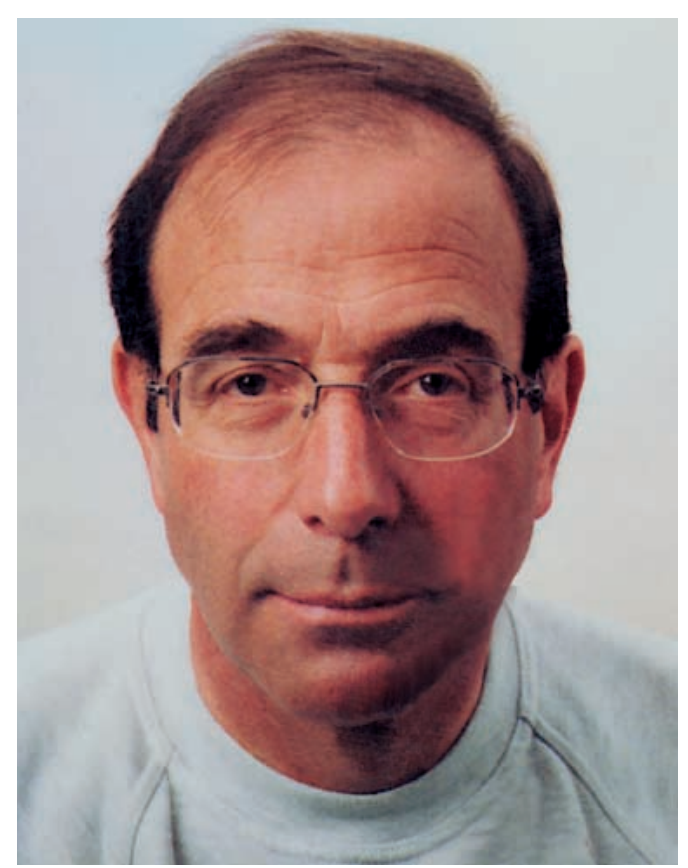

Woraus besteht Ihre Arbeit und wer hilft Ihnen dabei?

Ich bin vollamtlich angestellt und habe eine Kollegin an drei halben Tagen wöchentlich zur Seite sowie 280 Stellenprozente für Sachbearbeitung und Assistenz. Einen Stellvertreter habe ich nicht. Wichtig für meinen Beruf sind Kenntnisse in Public Health und Epidemiologie. Gefragt ist, die Fähigkeit zwischen verschiedenen Interessengruppen vermitteln zu können und Lösungen vorzubereiten. Dazu braucht es eine dicke Haut und eine gewisse Frustrationstoleranz, vor allem bei Reklamation nach Ablehnung von Gesuchen für ausserkantonale Hospitalisationen oder bei Budgetberatungen.

Meine wichtigsten Aufgaben sind:

- Einführungsgespräche mit allen neuen Ärztinnen und Ärzten vor der Praxiseröffnung.

- Überwachen der Infektionskrankheiten gemäss Meldeverordnung.

- Erteilen von Bewilligungen für Substitutionstherapien: methadon- und heroingestützte Behandlungen in drei Zentren.

- Beurteilen von Gesuchen für ausserkantonale Spitalbehandlungen gemäss KVG, etwa 7500 jährlich.

- Oberaufsicht über den schulärztlichen Dienst.

- Prävention und Gesundheitsförderung.

- Sanitätsdienstliche Versorgung der Bevölkerung in normalen und ausserordentlichen Lagen. Koordinierter Sanitätsdienst als Mitglied des kantonalen Führungsstabes.

- Mitarbeit bei der Qualitätssicherung in den 7 öffentlichen Spitälern. 
Ausserdem stehe ich der Regierung und der kantonalen Verwaltung als medizinische Fachperson zur Verfügung. Selbstverständlich bin ich auch für anrufende Bürgerinnen und Bürger für Auskünfte, Hilfe und Beratung erreichbar.

Gibt es so bei zahlreichen Partnern nicht auch Konflikte, zum Beispiel zwischen Kollegen und der Regierung oder grundsätzlich zwischen der angewandten und der verwalteten Medizin? Das wichtigste ist eine gute gegenseitige Information. Die Anliegen der Ärztegesellschaft sind im Gesundheitsdepartement viermal jährlich traktandiert. Ausserdem kommt es häufig zu Vernehmlassungen, und bei Geschäften, wie zum Beispiel der Reorganisation des schulärztlichen Dienstes, habe ich zusammen mit dem Präsidenten der Gesellschaft der Ärztinnen und Ärzte des Kantons Solothurn, Dr. med. Christoph Ramstein, das neue Konzept in vielen Gemeinden vorgestellt. Obwohl wir heute als Ärzte mehr hinterfragt werden als früher, finde ich mich im persönlichen Gespräch immer recht gut akzeptiert. Durch das Dauerthema der Finanzen hat es natürlich auch bei uns eine Verschiebung der Prioritäten auf die Seite der Ökonomie gegeben. Im eigentlichen Kernbereich der medizinischen Sachfragen hat sich hingegen nichts geändert. Es wird immer einen Übersetzer von der Medizin zur Verwaltung und umgekehrt brauchen.

Wie ist die Zusammenarbeit mit dem Bundesamt für Gesundheit und dessen Abteilungen?

Ich habe einen ausgezeichneten Kontakt zum BAG und ganz speziell zu einigen Mitarbeitern, die ausserordentlich kompetent sind. Die $\mathrm{Zu}-$ sammenarbeit ist sehr gut, rasch und effizient. Ich habe bisher alle Auskünfte immer prompt und korrekt erhalten. Auch hier ist es wichtig, dass ich mit den verschiedensten Abteilungen unkompliziert und ohne Formalitäten Kontakt aufnehmen kann. Es ist auch klar, dass das Bundesamt nicht immer alle Wünsche aus den Kantonen erfüllen kann. Gerne sähe ich eine einzige Ansprechperson im BAG, die für die interne Koordination der Bedürfnisse und Anregungen von Kantonsärzten zuständig wäre.

\section{Welches sind die Optionen und Chancen Ihres} Präsidentenamtes bei der VKS?

Als Präsident der Vereinigung der Kantonsärzte/ -ärztinnen der Schweiz habe ich sehr viel Kontakt mit den Kolleginnen und Kollegen aus anderen Kantonen. Ich habe die Chance, interessante und kompetente Leute kennenzulernen und auf viele Fachfragen direkt Einfluss zu nehmen. Zwar ist die Vereinigung privatrecht- lich organisiert, aber wir können trotzdem unsere Vorstellungen und Konzepte bei den Bundesbehörden einbringen. Bei mir sammeln sich viele Informationen aus den Kantonen an, die ich verarbeiten und als Synthese weitergeben kann, was sehr geschätzt wird. Dieses Präsidium gibt nicht viel Arbeit. Ich habe einen Vorstand, der mich unterstützt, und habe in der Privatindustrie gelernt, Sitzungen und Tagungen effizient zu organisieren und zu führen.

Alle Kantonsärzte haben mehr oder weniger die gleichen Sorgen. Wir haben untereinander ein ausgezeichnetes Verhältnis, ohne jeden Röstigraben, und schliesslich ist geteiltes Leid nur halbes Leid. Einmal im Jahr sitzen wir auch gerne einen ganzen Abend ungezwungen zusammen.

Wie eng arbeitet Solothurn mit den Nachbarkantonen zusammen, existieren regionale oder gar internationale Plattformen?

Diese Zusammenarbeit ist institutionalisiert. Mit Baselland haben wir ein Freizügigkeitsabkommen. Alle Kantonsbürgerinnen und -bürger können sich ohne Kostengutsprache in öffentlichen Spitälern behandeln lassen. Die beiden Kantone helfen sich auch gegenseitig bei der Bewältigung von ausserordentlichen Ereignissen oder Katastrophen. Auch mit Bern und dem Aargau arbeiten wir in der Spitalversorgung eng zusammen. International treffen wir uns aus der Nordostschweiz, dem Elsass und aus Baden an der Oberrheinkonferenz und koordinieren vor allem die Abwehr gegen Infektionskrankheiten.

\section{Wo engagieren Sie sich besonders gerne?}

Mir liegt viel daran, dass die Bevölkerung durch eine gute Durchimpfungsrate geschützt ist. Wir leben in einer Zeit, in der die meisten die Krankheiten wie Kinderlähmung, Diphtherie, Starrkrampf usw. nicht mehr kennen. Dadurch sind die Einwohner gegenüber Impfungen viel kritischer geworden. Sie werden dabei von falschen Informationen durch Impfgegner, unter denen leider auch Ärzte zu finden sind, irregeführt. Es wäre schade, wenn in der Schweiz durch verantwortungsloses Verhalten Krankheiten wie Kinderlähmung wieder auftauchen würden. Aus diesem Grund engagiere ich mich als Vizepräsident der Schweizerischen Gesellschaft für Impfpromotion SGIP für die Realisierung von kurzen, fachlich korrekten und leicht verständlichen Videos über Kinderkrankheiten, die durch Impfungen verhütet werden können. Auch an einem schulärztlichen Dienst liegt mir viel. Wir haben ein Konzept entwickelt, in dem der Schularzt eine zentrale Drehscheibenrolle in der Gesundheitsförderung und Prävention in der Schule 
übernimmt. Dazu gehören heute vor allem sozialmedizinische Aspekte, die in der Schule sehr gut angegangen werden können. Ein weiteres Steckenpferd von mir sind die von Zecken übertragenen Krankheiten.

Bitte einen Kommentar zum Gesundheitswesen? Gerne sähe ich eine Patentlösung für unsere Gesundheitsversorgung, die zu einem Selbstbedienungsladen geworden ist. Leider wird vielfach ein Lebensstil akzeptiert, dessen Folgen auf Kosten der Allgemeinheit repariert werden.

Die Einführung von sogenannten alternativen Methoden in die Grundversicherung finde ich falsch. Es handelt sich dabei um eine Zusatzmedizin, die in den Bereich der Privatversicherung gehört.
Ein Statement für die Leserinnen und Leser der Gelben?

Ich wünsche mir eine Rückkehr zum ärztlichen Kerngeschäft der Arzt-Patient-Beziehung, hin zur echten ärztlichen Tätigkeit, weg vom administrativen Ballast und den vielen nichtärztlichen Leistungen!

Herr Binz ich danke Ihnen für dieses Gespräch.

Interview: Dr. med. E. Taverna

progressiven Fahrers», dem eine Automarke wie ein Tierkreiszeichen zugeordnet wird. Die Wagentypen sind Lebensgefühl und Schicksal. Der BMW-Club Seetal bietet Grillparties und Chlauskegeln, VW-Käfer-Cabrio-Fahrerinnen und Ferrari-Piloten bilden eine verschworene Lebensgemeinschaft mit Ritualen und Stammeszeichen. Der Führerschein als Lebensdokument ist Initiation und Taufschein zur grossen Welt der Waschanlagen, der rasenden Gefühle auf der Autobahn und den freizeitfüllenden Shoppingtouren. Die ausgestellten Zubehöre mit den teuren Felgen und Spoilern, das Kino mit den alten Filmen von Sonntagsausflügen, sie wecken Erinnerungen an kribbelnde Picknicklust und Brechanfälle auf dem Hintersitz. Die Chronologien persönlicher Autokäufe und die Kinderträume vom echten grossen Brummer geraten zu intimeren Biografien als manche Psychoanalyse. «Weg von allem, weg von mir», weg von der Agglo Schweiz in die weite Welt hinaus. Über Kopfhörer und Videobilder kommen die Emotionen der Befragten ungefiltert zum Besucher. Eine Frau liegt im strohgefüllten Plastiksitz eines aufblasbaren Zeltes, wo die Sitzgruppen im Schummerlicht zum Zuhören einladen. Sie lauscht konzentriert den Hörbildern und Innenansichten von Autofrust und Fahrerlust. Aus diesen Erfahrungen haben die Autoren, der die Ausstellung begleitenden 


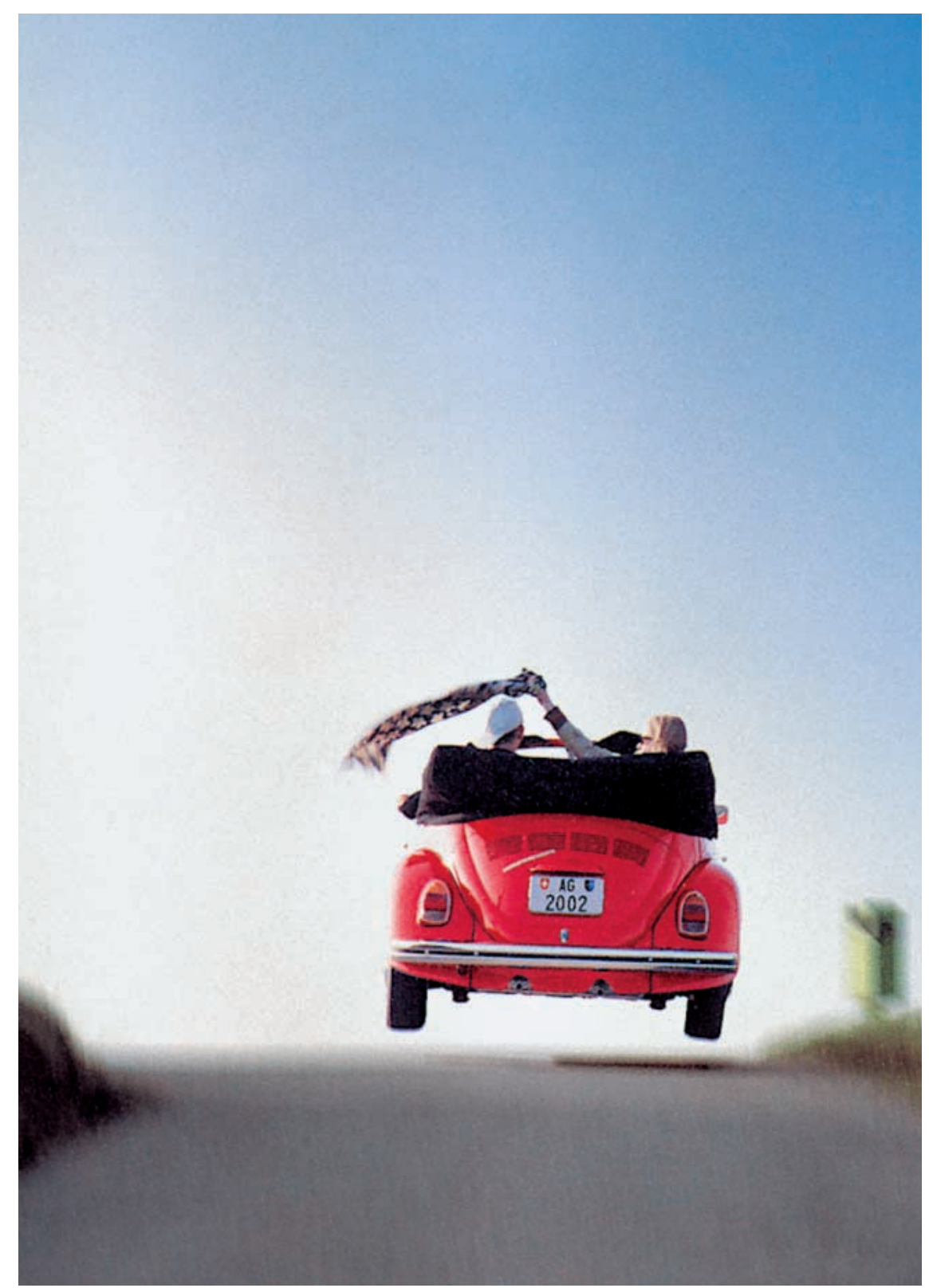

Lesungen, ihre Texte konzentriert, über «Schreibraser im Autostau» über die seltsamen Freiheiten der neuen Nomaden, über gewonnene Überholduelle, über Totschlag und dem Ende in der Radarfalle. Die Ausstellungsmacher haben die Themen und Räume verspielt und ironisch gestaltet. Da knirscht schwarzer Sand unter den Füssen und immer wieder neue Eingänge locken ins Innere von spiraligen und labyrinthischen Kammern, wo Informationen raffiniert in überraschende Ton- und Lichteffekte verpackt sind.

\section{... zum Frust}

Obwohl seit der letzten Eiszeit kein Ereignis unsere Landschaft dermassen verändert hat, werden die vielen Symptome dieser Lust ethnologisch-neutral, mit medizinischem Blick registriert. Manches hat keinen Platz, zum Beispiel das Auto als Liebeslaube und Todeszelle oder die zerquetschten Tiere und Fussgänger. Doch die Diagnosen sind auch so vielfältig genug und bleiben dem Besucher überlassen. Es gibt Szenen, die subversiv unter die Haut gehen, wenn zum Beispiel aus Notrufsäulen Reden zu Autobahnabschnittseröffnungen erklingen oder eine aufsteigende Rampe zu den unter Glas konservierten Zitaten von Philosophen und Schriftstellern führt. Sie sind in ihren Vitrinen wie Kassandra, treffsicher, prophetisch und wirkungslos. Wer noch bewusst die Nachkriegsjahre erlebte, weiss wie radikal und scheinbar unaufhaltsam unser Leben mit der Landschaft verändert wurde. Statistisch exakt wird die Verstädterung festgehalten. Der grosse Traum vom Eigenheim im Grünen wirft den Schatten einer Doppelgarage. Dafür steigt das Bruttosozialprodukt mit jedem Verletzten; alle 15 Stunden stirbt ein Mensch auf unseren Strassen. Es ist leichter, gegen das Rauchen Stimmung zu machen, als gegen den ebenso schädlichen Feinstaub vorzugehen, so wie es noch nicht üblich ist in den 7700 Staustunden jährlich die verlorenen Lebensjahre auszumachen. Das Phänomen hat seine blinden Flecken, seine politische Partei und Bundesräte, die pünktlich zum Automobilsalon reisen.

Die Stärke der Ausstellung des Stapferhauses Lenzburg ist die Reflexion über einen Gegenstand, den die Forscher fast so emotionslos wie einen Faustkeil aus der Steinzeit betrachten. Das Projekt sucht mit seiner Topografie der Autolust nach Klärungen zu Fragen wie: Was macht uns so immun gegen Erfahrungen wie Unglücksfälle, Lärm, Abgase, Parkplatzmangel und Verkehrskollaps? Was verrät diese obsessive Mobilmachung über unser privates, soziales und wirtschaftliches Leben? Die SBB unterstützen das Nachdenken. Das Areal liegt nur zehn Gehminuten vom Bahnhof entfernt.

\section{Auskunft}

Stapferhaus Lenzburg, Schloss, 5600 Lenzburg 2, Tel. 06288848 00, E-Mail: info@stapferhaus.ch oder www.stapferhaus.ch 